\title{
Analysis of laser-microdissected prostate cancer tissues reveals potential tumor markers
}

\author{
DAVID ADLER ${ }^{*}$, ANDREAS LINDSTROT ${ }^{*}$, REINHARD BUETTNER and NICOLAS WERNERT \\ Institute of Pathology, University Hospital Bonn, D-53127 Bonn, Germany
}

Received April 18,2011; Accepted June 2, 2011

DOI: $10.3892 / \mathrm{ijmm} .2011 .746$

\begin{abstract}
Prostate cancer (PCA) is a clinically heterogeneous and often multifocal disease with a clinical outcome difficult to predict. A deeper knowledge of the molecular basis of the disease may lead to a better prediction of prognosis. Therefore, in this study we investigated the molecular basis of PCA by identifying potential tumor markers in laser-microdisected PCA tissues. Among a group of PCA patients, quantitative RT-PCR analysis was performed to compare the expression of 70 genes. These genes were selected from the results of two microarrays which investigated the gene expression profile differences between moderately or poorly differentiated prostate carcinoma glands and the corresponding normal glands. Among the genes examined, CDKN2A, GATA3, CREBBP, ITGA2, NBL1 and TGM4 were down-regulated in the prostate carcinoma glands compared to the corresponding normal glands, whereas TFF3, TMPRSS2 and ERG were up-regulated. Our findings indicate that these genes may play roles as tumor suppressor genes or oncogenes in PCA, and may serve as potential tumor markers and novel therapeutic targets.
\end{abstract}

\section{Introduction}

Prostate cancer (PCA) is one of the most common cancers in the Western world (1). As a result of both steadily increasing age of western populations and the introduction of systematic prostate specific antigen (PSA) screening since 1987, PCA incidence is steadily increasing (2,3). A major problem with PCA is its clinical heterogeneity. PCAs include both indolent carcinomas carrying a favorable prognosis and requiring just watchful waiting, and aggressive tumors with distant metastases and a necessary systemic treatment (4-6). Based

Correspondence to: Professor Nicolas Wernert, Institute of Pathology, University Hospital Bonn, Sigmund-Freud-Str. 25, D-53127 Bonn, Germany

E-mail: nicolas.wernert@ukb.uni-bonn.de

*Contributed equally

Key words: transcriptional regulation, prostate cancer, microarray upon clinical and biopsy-findings alone, it is not possible to exactly predict the outcome of a PCA in individual patients (3). Therefore, considerable research efforts have been undertaken to identify molecular markers carrying independent prognostic information or serving as potential therapeutic targets $(5,6)$.

As PCA is considered to be a clinically heterogeneous and often multifocal disease with a clinical outcome that is difficult to predict $(7,8)$, an understanding of the molecular events of the disease may improve prediction of prognosis, as genetic aberrations drive the formation and aggressiveness of prostate carcinoma (9). In an attempt to understand these molecular events, a notable number of studies have investigated the roles that epigenetics, oncogene/tumor suppressors, androgen signaling and microRNAs (miRNAs) may play in PCA (10-42).

Epigenetic studies deal with the modifications of gene expression without alterations of DNA sequences (43). There are two epigenetic strategies, the direct methylation of the so-called $\mathrm{CpG}$ islands (cystins followed by guanosines preferably in the promotor regions of genes) $(44,45)$ and the modification of histones (43). The modifications of histones include acetylation, ADP-ribosylation, ubiquination, phosphorylation and methylation $(43,45)$. The degree of methylation in histones can be mono- to trimethylation and depending on the position of the modification, the gene can be silenced or transcribed (44). It has been proposed that every cancer has its own histone map (28). DNA methylation and histone modifications have been linked to prostatic carcinogenesis, and are suggested to influence tumor formation (10). However, the reasons that lie behind these epigenetic changes remain to be investigated, even though external influences such as diet and oxidative stress are suggested to be involved $(10,46)$.

Both tumor suppressors and oncogenes are known to contribute to the carcinogenic process and to tumor development (11). In PCA, both tumor suppressors and oncogenes have been found, however genes that are uniquely involved in prostate-specific carcinogenesis have not yet been identified (11). The RAS gene family of oncogenes is reported to be activated up to $50 \%$ in human cancers, whereas it seems to have only a minor role in the development of PCA $(32,38)$. On the other hand, tumor suppressors such as the p53 protein encoding gene, which regulates cell cycle arrest and apoptosis, has a low mutation frequency in early PCA, whereas a heterozygous loss of function mutations are often associated with the late stage (33). Other tumor suppressor genes such the 
Table I. The sequences of primers used in the qRT-PCR analysis.

\begin{tabular}{|c|c|c|}
\hline Name & Left & Right \\
\hline$C D K N 2 A$ & TGCCTTTTCACTGTGTTGGA & GAGCTTTGGTTCTGCCATTT \\
\hline$C R E B B P$ & GGATGATCACTCTTAGACCATGC & CCACCCTTTTTGTCTGTTG \\
\hline$E R G$ & GACGACTTCCAGAGGCTCAC & ATAAAAGCTGCACCCCCTGT \\
\hline GATA3 & GTCCTGTGCGAACTGTCAGA & GGGGAAGTCCTCCAGTGAGT \\
\hline ITGA2 & GCAGCTGCAGAAATCAACAC & CCGAGCTTCCATAAAATTGC \\
\hline$N B L 1$ & СТСТТССТССССТСТСАТСС & AAATTGTGCAGCTTGGCTCT \\
\hline rPL13A (control) & TACGCTGTGAAGGCATCAAC & CACCATCCGCTTTTTCTTGT \\
\hline TFF3 & AGTGCCTTGGTGTTTCAAGC & GAGCATGGGACCTTTATTCG \\
\hline TGM4 & GCACCCCAATAAAAACTGGA & TTTTTGACCTGCTGGCTCTT \\
\hline TMPRSS2 & GGCCTATTTCAGCTGCTTTG & GGAGGCAGAACCATGGTAGA \\
\hline
\end{tabular}

Glioma pathogenesis-related protein1 (GLIPRI), and PTEN have been found to be down-regulated in PCA, to be involved in p53-dependent and independent apoptotic pathways and to be associated with a poor clinical outcome, respectively $(30,37,39,41,42)$.

Normal and neoplastic growth of the prostate gland depends on the androgen receptor (AR) (14). The AR is crucial for proliferation and progression of PCA $(12,13)$. However the androgen-independent growth mechanisms are not yet well understood (14). It has been reported that the $A R$ may be amplified and overexpressed $(15,16)$, hypersensitive to androgens or activated by non-androgenic ligands in androgen-independent PCA growth (17).

miRNAs are small noncoding RNAs which target the mRNAs for cleavage or translational repression (47). In PCA, various miRNAs were found to be underexpressed or overexpressed suggesting that miRNAs may be involved in PCA development as well (27). Furthermore, miRNAs can act as onco-miRNAs (e.g. miR-21, -125b, -221/222) (35,36,40), inhibiting the translation of tumor suppressor genes, or as tumor suppressor miRNAs (e.g. miR-101, -126*, -146a, -200 family, -330) $(31,34,48-50)$, inhibiting oncogenes (29).

In addition to efforts investigating the role that epigenetics, oncogene/tumor suppressors, androgen signaling and miRNAs may play in PCA, recent studies have focused on investigating translocations in PCA between the TMPRSS 2 gene encoding an androgen-regulated prostate-specific serine protease and several genes of the ETS family of transcription factors, most commonly ERG (51-54). These gene fusions are reported in up to $60-70 \%$ of clinically manifested PCAs (10), and it is known that such translocations lead to an increased expression of the rearranged ETS factors in response to androgens (52). Furthermore, the ETS family members were initially viewed as oncogenic factors, but recently, they have been suggested to play roles as tumor- or metastasis-suppressors as well (55).

In this study, we have attempted to investigate the molecular basis of PCA by identifying potential tumor markers. We report here the identification of a set of genes in PCA tissues, which may function as tumor suppressors or oncogenes. These identified genes may serve as potential markers for early tumor detection, and therapeutic drug treatment.

\section{Materials and methods}

Processing of human prostatectomy specimens. Fresh tissue samples out of the peripheral zone (prostate proper, sample sizes around $0.5 \times 0.5 \times 0.3 \mathrm{~cm}$ ) from 11 prostate carcinoma patients were taken immediately after radical prostatectomy and shock-frozen in liquid nitrogen with ice-cold isopentane. Frozen sections $(6 \mu \mathrm{m})$ were cut from the samples using a cryotome (Leica, Germany) and mounted on membrane-coated slides (MembraneSlides, $1 \mathrm{~mm}$ PEN, Zeiss, Germany) for subsequent laser-microdissection. One section was mounted on conventional slides and stained with hematoxylin and eosin (H\&E) for diagnostic evaluation by an experienced pathologist. Laser-microdissection was performed according to our previously published method (56-58). Frozen sections were dried for $2 \mathrm{~min}$ in the cryotome, washed for $2 \mathrm{~min}$ with $70 \%$ ethanol in DEPC-treated water and stained for $30 \mathrm{sec}$ in $1 \%$ Cresyl Violet diluted in 50\% ethanol-DEPC-treated water. Slides were then washed briefly in 70 and $100 \%$ ethanol, dried for $10 \mathrm{~min}$ and stored at $-80^{\circ} \mathrm{C}$ until use for laser-microdissection.

Quality control. A frozen section from each patient prior to laser-capture microdissection (LCM) and the dissected slide after LCM were used for quality control. For RNA extraction with the RNeasy mini kit (Qiagen, Germany) the section was washed from the slide with $600 \mu 12 \mu \mathrm{M}$ DTT in RLT buffer and vortexted for $30 \mathrm{sec}$. The recommend DNase digestion was carried out with an RNase-free DNase Set (Qiagen). RNA integrity was measured with the Agilent Bioanalyzer 2100 (Agilent Technologies, USA). Samples with a RIN factor $>6$ were used for laser-capture microdissection and microarray.

Laser-capture microdissection. Laser-capture microdissection of Cresyl Violet-stained sections was performed using the Axio Observer. Z1 microscope (Zeiss) with the integrated Palm MicroBeam software (Zeiss). LCM was performed under a x10 objective lens. Isolated normal or cancerous glands were collected in $200 \mu \mathrm{l}$ adhesive cap tubes (Zeiss).

Microarray analysis of RNA isolated from laser microdisected moderately and poorly differentiated prostate carcinoma 
CDKN2A

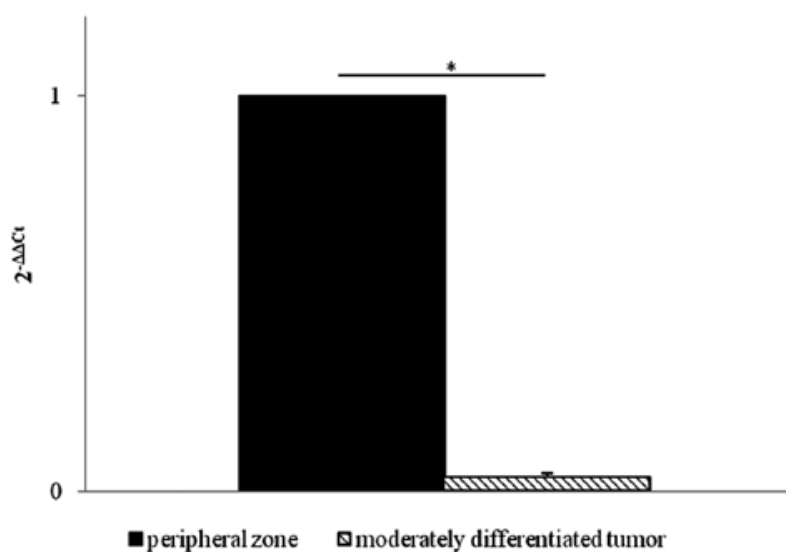

Figure 1. Expression of $C D K N 2 A$. The relative expression of $C D K N 2 A$ as measured by quantitative RT-PCR in the moderately differentiated prostate carcinoma glands compared to the normal peripheral zone glands. Significance was calculated with the Student's t-test; ${ }^{*} \mathrm{P}<0.05$. glands, as well as from normal glands of prostate cancer patients. RNA was isolated using the RNeasy micro kit (including the recommend DNase digestion with the RNasefree DNase set, Qiagen) from laser microdissected moderately and poorly differentiated prostate carcinoma glands, as well as from the normal prostate proper glands of prostatectomy specimens. The quantity of RNA was measured using the NanoDrop photometer (Thermo Fisher Scientific, USA). Thereafter, an equal amount of RNA from the moderately differentiated glands, poorly differentiated glands, and normal peripheral glands were pooled to final concentrations of $300 \mathrm{ng}$. The microarray analysis and bioinformatical interpretation were performed at Miltenyi Biotec (Germany) with the Whole Human Genome Oligo Microarray 4x44K (Agilent, USA). The microarray results were validated in a subset of genes by quantitative RT-PCR (qRT-PCR).

Quantitative RT-PCR using RNA isolated from laser microdisected moderately and poorly differentiated prostate carcinoma glands, as well as from normal glands of prostate cancer patients. qRT-PCR was performed by comparing the expression of 70 genes that were selected from the microarrays in the moderately or poorly differentiated carcinoma glands to the corresponding normal glands of each of the 11 PCA patients. Briefly, following the reverse transcription of totalRNA with SuperScript III First-Strand Synthesis SuperMix for qRT-PCR (Invitrogen, USA), the quantitative real-time PCR was performed using SYBR-GreenER qPCR SuperMix (Invitrogen, USA) in a total volume of $10 \mu \mathrm{l}$. The PCR conditions in the Applied Biosystems 7900HT instrument were as follows: $2 \min 50^{\circ} \mathrm{C}, 10 \min 95^{\circ} \mathrm{C}, 40$ cycles $15 \mathrm{sec} 95^{\circ} \mathrm{C}$ and $1 \mathrm{~min} 60^{\circ} \mathrm{C}$. At the end, a melting curve analysis with $15 \mathrm{sec}$ $95^{\circ} \mathrm{C}, 15 \mathrm{sec} 60^{\circ} \mathrm{C}$ and $15 \mathrm{sec} 95^{\circ} \mathrm{C}$ was performed. The data were collected after the $60^{\circ} \mathrm{C}$ step at every cycle and after the melting point analysis. The primers of the genes that were used in the qRT-PCR analysis and were found to be significantly differentially expressed in the normal peripheral zone glands compared to the moderately or poorly differentiated carcinoma glands are listed in Table I.
GATA3

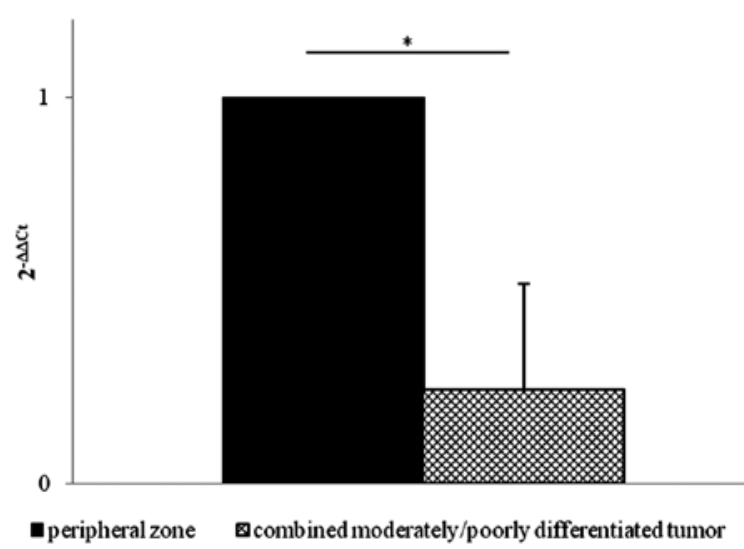

Figure 2. Expression of GATA3. The relative expression of GATA3 as measured by quantitative RT-PCR in combined moderately/poorly differentiated prostate carcinoma glands compared to the normal peripheral zone glands. Significance was calculated with the Student's t-test; ${ }^{*} \mathrm{P}<0.05$.

\section{Results}

Gene expression analysis of moderately and poorly differentiated prostate carcinoma glands compared to normal glands of the prostate proper (or the peripheral zone) from PCA patients using Whole Human Genome Oligo microarrays. To identify potential tumor gene markers in PCA, we analyzed the gene expression profile in the moderately and poorly differentiated prostate carcinoma glands compared to normal glands of the peripheral zone from PCA patients using two Whole Human Genome Oligo microarrays. The two microarrays yielded 8,242 and 6,215 differentially expressed genes with a fold change $>2$ and a $\mathrm{P}$-value $<0.01$ in the normal peripheral zone compared to the moderately and poorly differentiated prostate carcinoma glands, respectively (data not shown). A clustering analysis based on gene function placed each gene into one or more of following categories: oncogenes, tumor suppressors, apoptosis, AR pathway, development, angiogenesis, immunity genes, as well uncategorized genes (data not shown).

qRT-PCR analysis of selected genes in the moderately or poorly differentiated carcinoma glands compared to the corresponding normal peripheral zone glands of PCA patients. Due to the extremely limited amounts of RNA that can be extracted from PCA tissues of each of the 11 prostate carcinoma patients, we chose 70 highly differentially expressed genes from the various categories provided by the microarray, and examined their expression in each of the 11 patients by qRT-PCR. Among the 70 genes examined, 9 genes were found to be significantly differentially expressed in the normal peripheral zone glands compared to the moderately or poorly differentiated carcinoma glands (Figs. 1-8). Our data show that the genes CDKN2A, GATA3, CREBBP, ITGA2, NBL1 and TGM4 are down-regulated in the moderately or poorly differentiated carcinoma glands compared to the normal peripheral zone glands (Figs. 1-6). On the other hand, the genes TMPRSS2, $E R G$ and TFF3 are up-regulated in moderately or poorly differentiated carcinoma glands compared to the normal peripheral zone glands (Figs. 7 and 8). 


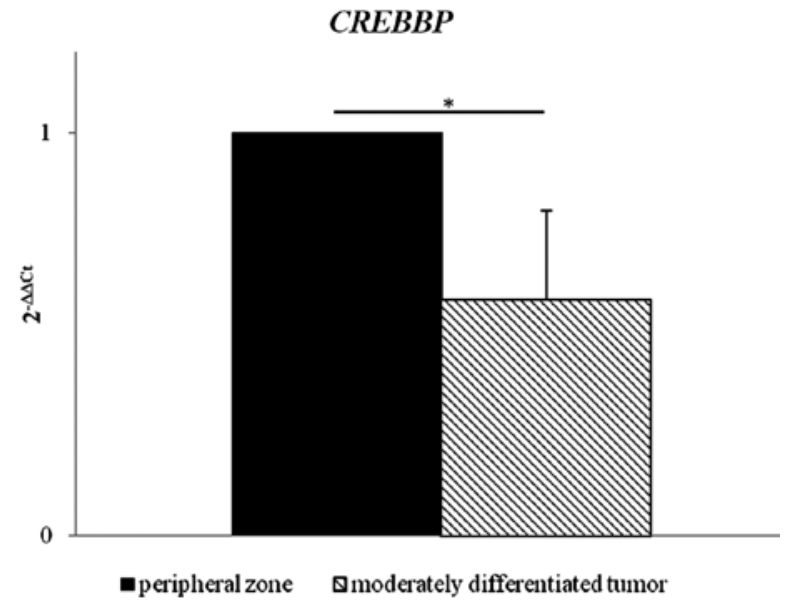

Figure 3. Expression of $C R E B B P$. The relative expression of $C R E B B P$ as measured by quantitative RT-PCR in the moderately differentiated prostate carcinoma glands compared to the normal peripheral zone glands. Significance was calculated with the Student's t-test. P-values indicated as " $\mathrm{P}<0.05$.

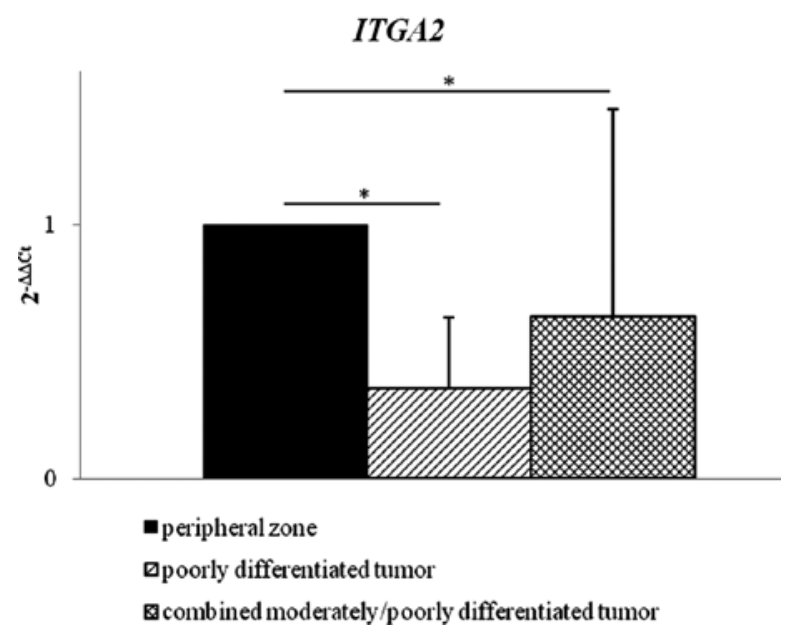

Figure 4. Expression of ITGA2. The relative expression of ITGA2 as measured by quantitative RT-PCR in the poorly and combined moderately/poorly differentiated prostate carcinoma glands compared to the normal peripheral zone glands. Significance was calculated with the Student's t-test. P-values indicated as ${ }^{*} \mathrm{P}<0.05$

\section{Discussion}

Tumor development and progression involve changes in gene expression patterns in various pathways $(59,60)$. Genes involved in such pathways may be classified into various categories, such as oncogenes, tumor suppressor genes, apoptosis-relevant genes or DNA-repair genes (60-63). PCA is a clinically heterogeneous and often multifocal disease with a clinical outcome difficult to predict $(7,8)$. As genetic aberrations drive the formation and aggressiveness of prostate carcinoma, a knowledge of PCA at the molecular level may improve our understanding of the disease and lead to a better prediction of prognosis (9).

Therefore, in an attempt to identify potential tumor markers, in this study we investigated the gene expression profile in the prostate carcinoma glands compared to normal glands of the peripheral zone from PCA patients using microarrays and qRT-PCR analysis.

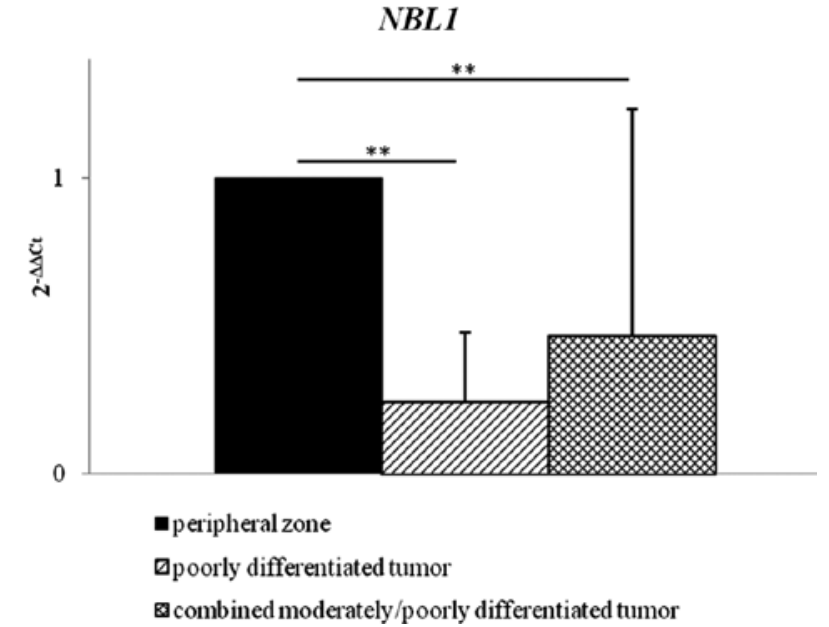

Figure 5. Expression of $N B L 1$. The relative expression of $N B L 1$ as measured by quantitative RT-PCR in the poorly and combined moderately/poorly differentiated prostate carcinoma glands compared to the normal peripheral zone glands. Significance was calculated with the Student's t-test. P-values indicated as ${ }^{* *} \mathrm{P}<0.01$

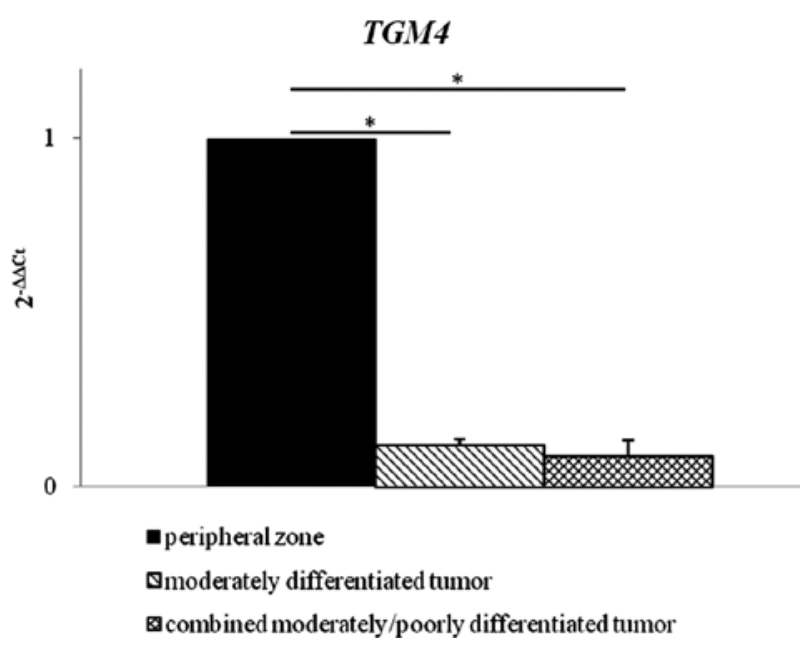

Figure 6. Expression of TGM4. The relative expression of TGM4 as measured by quantitative RT-PCR in the moderately and combined moderately/poorly differentiated prostate carcinoma glands compared to the normal peripheral zone glands. Significance was calculated with the Student's t-test; ${ }^{\text {P }}<0.05$.

We report here the identification of 9 genes in PCA tissues including the well-known genes TMPRSS2 and $E R G$, which may function as tumor suppressor genes, oncogenes, or apoptosis-related genes, and which may serve as potential tumor markers (Figs. 1-8).

The genes CDKN2A, GATA3, CREBBP, ITGA2, NBL1 and $T G M 4$ were found to be down-regulated in prostate carcinoma glands compared to the normal peripheral zone glands, indicating that these genes may function as tumor suppressors in PCA (Figs. 1-6). On the other hand, the genes TFF3, TMPRSS2 and $E R G$ were found to be up-regulated in the prostate carcinoma glands compared to the normal peripheral zone glands, indicating that these genes may play the role of oncogenes in PCA (Figs. 7 and 8).

Briefly, CDKN2A which has been found to be downregulated in the moderately differentiated carcinoma glands compared to the normal peripheral zone glands (Fig. 1), is 


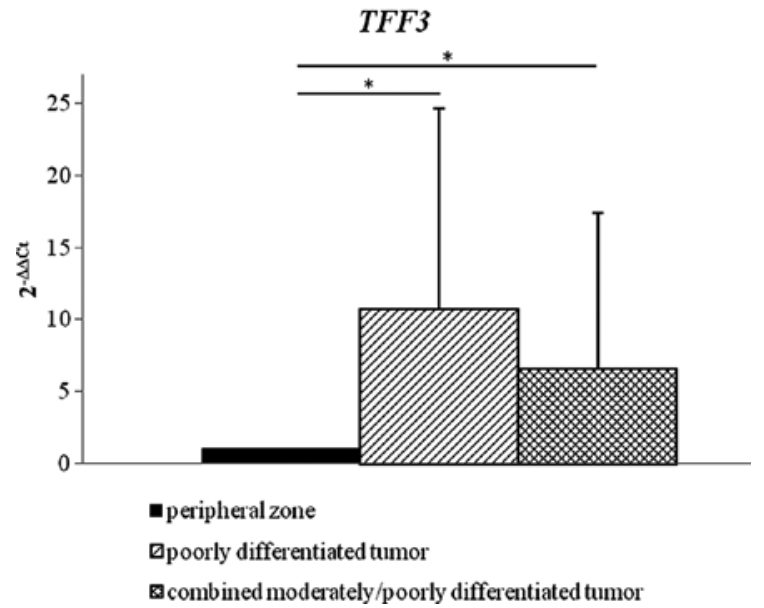

Figure 7. Expression of TFF3. The relative expression of TFF3 as measured by quantitative RT-PCR in the poorly and combined moderately/poorly differentiated prostate carcinoma glands compared to the normal peripheral zone glands. Significance was calculated with the Student's t-test; ${ }^{*} \mathrm{P}<0.05$.

reported to be a tumor suppressor gene $(64,65) . C D K N 2 A$ is known to be inactivated in most melanoma cell lines (66), and it is suggested that families carrying a $C D K N 2 A$ mutation have an increased risk of multiple melanomas, pancreatic carcinoma, and breast cancer (65). Furthermore, inactivation of $C D K N 2 A$ is implicated in primary central nervous system lymphomas (PCNSL) (67). Therefore, our finding that $C D K N 2 A$ is down-regulated in PCA tissues suggests a role of $C D K N 2 A$ as a potential tumor suppressor gene in PCA. The gene GATA3 which is found to be down-regulated in the prostate carcinoma glands compared to the normal peripheral zone glands (Fig. 2) is reported to be involved in the androgen regulation of the PSA gene (68). GATA3 is expressed at low levels in invasive breast carcinomas and suggested to be a potential breast cancer prognostic marker (69). The reported low expression of GATA3 in breast carcinomas is similar to our findings in prostate carcinoma, indicating that GATA3 may function as a tumor suppressor in both breast and PCA. The CREBBP gene has been found to be mutated in human cancers and encodes a protein which is reported to be a tumor suppressor protein, that is essential in cell cycle control, differentiation and development (70). We found the CREBBP gene to be down-regulated in the moderately differentiated prostate carcinoma glands compared to the normal peripheral zone glands (Fig. 3). Our findings thus support the role of CREBBP as a tumor suppressor, and indicate that it may function as a tumor suppressor in PCA as well. ITGA2 which is found to be down-regulated in the poorly differentiated prostate carcinoma glands compared to the normal peripheral zone glands (Fig. 4), has been reported to be down-regulated in breast cancer tissues as well (71). These findings indicate that ITGA2 is very likely to play the role of a tumor suppressor in PCA. NBL1 which is found to be down-regulated in the poorly differentiated prostate carcinoma glands compared to the normal peripheral zone glands (Fig. 5), has been suggested as a possible tumor suppressor gene of human neuroblastoma (72). Furthermore, electronic profiling of expressed sequence tags and qRT-PCR have reported that the expression of NBL1 is reduced by over $80 \%$ in PCA (73). These previously reported studies support our findings and the notion that $N B L 1$ may play the role of a tumor suppressor in PCA. TGM4 tissue-specifically expressed in the prostate (74), is found to be down-regulated in the moderately differentiated carcinoma glands compared to the normal peripheral zone glands (Fig. 6). Studies of normal prostate and human prostate carcinoma reported TGM4 expression in the normal prostate, but not in PCA (75). Furthermore, TGM4 expression was not detected in the human PCA cell lines, LNCaP and PC3 (74). These previous studies combined with our findings may indicate a possible role of TGM4 as a tumor suppressor gene in PCA.

On the contrary, the genes TFF3, TMPRSS 2 and $E R G$ were found to be up-regulated in the prostate carcinoma glands compared to the normal peripheral zone glands (Figs. 7 and 8), respectively. In agreement with our findings (Fig. 7), TFF3 was reported to be overexpressed in PCA (76). Interestingly, $E R G$ which is found to be up-regulated in the prostate carcinoma glands (Fig. 8A) is reported to be implicated in the regulation of TFF3 in PCA progression (77). As TMPRSS2 is already known to be overexpressed in PCA (78), and since translocations between TMPRSS2 and several members of the ETS family, most commonly $E R G$, are frequently found in PCA (51-54),
A

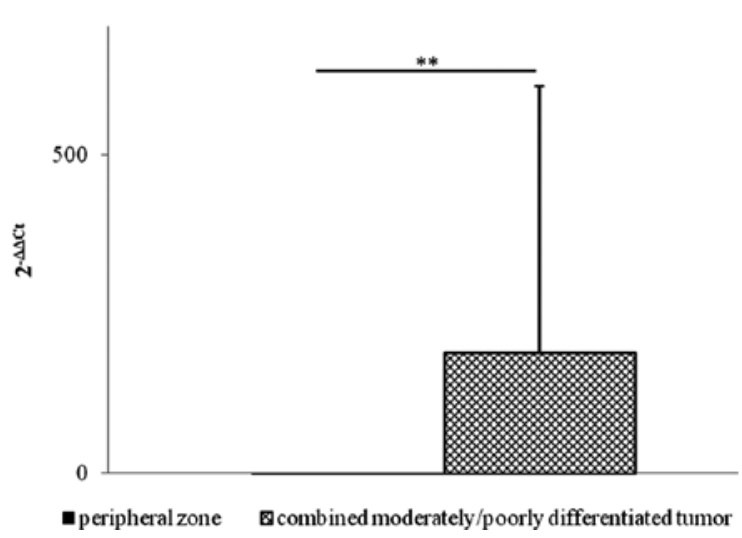

B

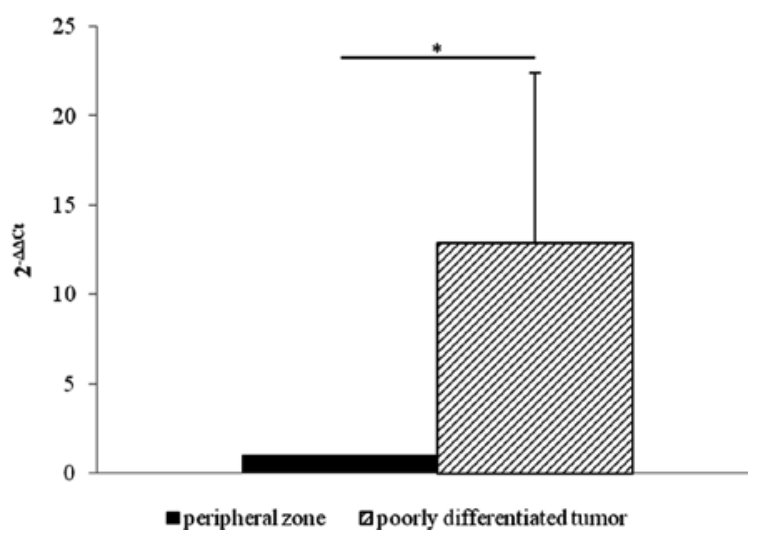

Figure 8. Expression of ERG and TMPRSS2. The relative expression of (A) ERG and (B) TMPRSS2 as measured by quantitative RT-PCR in the combined moderately/poorly differentiated, and moderately differentiated prostate carcinoma glands, respectively, compared to the normal peripheral zone glands. Significance was calculated with the Student's t-test. P-values indicated as ${ }^{* *} \mathrm{P}<0.01$ and ${ }^{*} \mathrm{P}<0.05$. 
we investigated the expression of both $E R G$ and TMPRSS 2 in our prostate carcinomas as well. Our findings that TMPRSS2 and $E R G$ are up-regulated in the prostate carcinoma glands compared to the normal peripheral zone glands (Fig. 8) further supports the previously reported studies, and may indicate a possible role of these genes as oncogenes in PCA.

Taken together, our analysis of laser-microdissected PCA tissues revealed a set of genes which may function as tumor suppressor genes or oncogenes in PCA. These genes may serve as potential tumor markers and novel therapeutic targets. Furthermore, investigating such candidate genes may add to connect state of knowledge of the molecular basis of PCA.

\section{Acknowledgements}

We are grateful to the DFG(Deutsche Forschungsgemeinschaft; German Research Association, grant no. WE 1104/11-1) and the Deutsche Krebshilfe German Cancer Aid, grant no. 107827) for financial support.

\section{References}

1. Jemal A, Siegel R, Xu J and Ward E: Cancer statistics, 2010. CA Cancer J Clin 60: 277-300, 2010.

2. Draisma G, Etzioni R, Tsodikov A, et al: Lead time and overdiagnosis in prostate-specific antigen screening: importance of methods and context. J Natl Cancer Inst 101: 374-383, 2009.

3. Park JY: Promoter hypermethylation in prostate cancer. Cancer Control 17: 245-255, 2010.

4. Wu JN and Dall'Era MA: Active surveillance for localized prostate cancer - current practices and recommendations. Sci World J 10: 2352-2361, 2010

5. Liang Y, Ankerst DP, Ketchum NS, et al: Prospective evaluation of operating characteristics of prostate cancer detection biomarkers. J Urol 185: 104-110, 2011.

6. Thompson IM, Pauler DK, Goodman PJ, et al: Prevalence of prostate cancer among men with a prostate-specific antigen level $<$ or =4.0 ng per milliliter. N Engl J Med 350: 2239-2246, 2004.

7. Arora R, Koch MO, Eble JN, Ulbright TM, Li L and Cheng L: Heterogeneity of Gleason grade in multifocal adenocarcinoma of the prostate. Cancer 100: 2362-2366, 2004.

8. Ruijter ET, van de Kaa CA, Schalken JA, Debruyne FM and Ruiter DJ: Histological grade heterogeneity in multifocal prostate cancer. Biological and clinical implications. J Pathol 180: 295-299, 1996.

9. DeMarzo AM, Nelson WG, Isaacs WB and Epstein JI: Pathological and molecular aspects of prostate cancer. Lancet 361: 955-964, 2003.

10. Joshua AM, Evans A, Van der Kwast T, et al: Prostatic preneoplasia and beyond. Biochim Biophys Acta 1785: 156-181, 2008.

11. Isaacs W and Kainu T: Oncogenes and tumor suppressor genes in prostate cancer. Epidemiol Rev 23: 36-41, 2001.

12. Feldman BJ and Feldman D: The development of androgenindependent prostate cancer. Nat Rev Cancer 1: 34-45, 2001.

13. Balk SP: Androgen receptor as a target in androgen-independent prostate cancer. Urology 60 (Suppl 1): S132-S139, 2002.

14. Koochekpour S: Androgen receptor signaling and mutations in prostate cancer. Asian J Androl 12: 639-657, 2010.

15. Visakorpi T, Hyytinen E, Koivisto $\mathrm{P}$, et al: In vivo amplification of the androgen receptor gene and progression of human prostate cancer. Nat Genet 9: 401-406, 1995.

16. Brown RS, Edwards J, Dogan A, et al: Amplification of the androgen receptor gene in bone metastases from hormonerefractory prostate cancer. J Pathol 198: 237-244, 2002.

17. Grossmann ME, Huang H and Tindall DJ: Androgen receptor signaling in androgen-refractory prostate cancer. J Natl Cancer Inst 93: 1687-1697, 2001.

18. Cummins JM,He Y,Leary RJ, et al: The colorectal microRNAome. Proc Natl Acad Sci USA 103: 3687-3692, 2006.

19. Iorio MV, Ferracin M, Liu CG, et al: MicroRNA gene expression deregulation in human breast cancer. Cancer Res 65: 7065-7070, 2005 .
20. Yanaihara N, Caplen N, Bowman E, et al: Unique microRNA molecular profiles in lung cancer diagnosis and prognosis. Cancer Cell 9: 189-198, 2006.

21. Calin GA, Ferracin M, Cimmino A, et al: A MicroRNA signature associated with prognosis and progression in chronic lymphocytic leukemia. N Engl J Med 353: 1793-1801, 2005.

22. Volinia S, Calin GA, Liu CG, et al: A microRNA expression signature of human solid tumors defines cancer gene targets. Proc Natl Acad Sci USA 103: 2257-2261, 2006.

23. He H, Jazdzewski K, Li W, et al: The role of microRNA genes in papillary thyroid carcinoma. Proc Natl Acad Sci USA 102: 19075-19080, 2005.

24. Murakami Y, Yasuda T, Saigo K, et al: Comprehensive analysis of microRNA expression patterns in hepatocellular carcinoma and non-tumorous tissues. Oncogene 25: 2537-2545, 2006.

25. Lu J, Getz G, Miska EA, et al: MicroRNA expression profiles classify human cancers. Nature 435: 834-838, 2005.

26. Mattie MD, Benz CC, Bowers J, et al: Optimized high-throughput microRNA expression profiling provides novel biomarker assessment of clinical prostate and breast cancer biopsies. Mol Cancer 5: 24, 2006.

27. Porkka KP,Pfeiffer MJ, Waltering KK, Vessella RL, Tammela TL and Visakorpi T: MicroRNA expression profiling in prostate cancer. Cancer Res 67: 6130-6135, 2007.

28. Esteller M: Epigenetics in cancer. N Engl J Med 358: 1148-1159, 2008.

29. Heneghan HM, Miller N and Kerin MJ: MiRNAs as biomarkers and therapeutic targets in cancer. Curr Opin Pharmacol 10: 543-550, 2010.

30. Kim S, Huang W, Mottillo EP, et al: Posttranslational regulation of membrane type 1-matrix metalloproteinase (MT1-MMP) in mouse PTEN null prostate cancer cells: enhanced surface expression and differential O-glycosylation of MT1-MMP. Biochim Biophys Acta 1803: 1287-1297, 2010.

31. Kong D, Li Y, Wang Z, et al: miR-200 regulates PDGF-Dmediated epithelial-mesenchymal transition, adhesion, and invasion of prostate cancer cells. Stem Cells 27: 1712-1721, 2009.

32. Kranenburg O: The KRAS oncogene: past, present, and future. Biochim Biophys Acta 1756: 81-82, 2005.

33. Lee JT, Lehmann BD, Terrian DM, et al: Targeting prostate cancer based on signal transduction and cell cycle pathways. Cell Cycle 7: 1745-1762, 2008.

34. Lee KH, Chen YL, Yeh SD, et al: MicroRNA-330 acts as tumor suppressor and induces apoptosis of prostate cancer cells through E2F1-mediated suppression of Akt phosphorylation. Oncogene 28: 3360-3370, 2009.

35. Lee YS, Kim HK, Chung S, Kim KS and Dutta A: Depletion of human micro-RNA miR-125b reveals that it is critical for the proliferation of differentiated cells but not for the downregulation of putative targets during differentiation. J Biol Chem 280: 16635-16641, 2005.

36. Li T, Li D, Sha J, Sun P and Huang Y: MicroRNA-21 directly targets MARCKS and promotes apoptosis resistance and invasion in prostate cancer cells. Biochem Biophys Res Commun 383: 280-285, 2009.

37. McCall P, Witton CJ, Grimsley S, Nielsen KV and Edwards J: Is PTEN loss associated with clinical outcome measures in human prostate cancer? Br J Cancer 99: 1296-1301, 2008.

38. Pearson HB, Phesse TJ and Clarke AR: K-ras and Wnt signaling synergize to accelerate prostate tumorigenesis in the mouse. Cancer Res 69: 94-101, 2009.

39. Ren C, Ren CH, Li L, Goltsov AA and Thompson TC: Identification and characterization of RTVP1/GLIPR1-like genes, a novel p53 target gene cluster. Genomics 88: 163-172, 2006.

40. Sun T, Wang Q, Balk S, Brown M, Lee GS and Kantoff P: The role of microRNA-221 and microRNA-222 in androgen-independent prostate cancer cell lines. Cancer Res 69: 3356-3363, 2009.

41. Thompson TC: Glioma pathogenesis-related protein 1: tumorsuppressor activities and therapeutic potential. Yonsei Med J 51: 479-483, 2010.

42. Yoshimoto M, Cunha IW, Coudry RA, et al: FISH analysis of 107 prostate cancers shows that PTEN genomic deletion is associated with poor clinical outcome. Br J Cancer 97: 678-685, 2007.

43. Costa FF: Epigenomics in cancer management. Cancer Manag Res 2: 255-265, 2010.

44. Iacobuzio-Donahue CA: Epigenetic changes in cancer. Annu Rev Pathol 4: 229-249, 2009. 
45. Perry AS, Watson RW, Lawler $M$ and Hollywood D: The epigenome as a therapeutic target in prostate cancer. Nat Rev Urol 7: 668-680, 2010.

46. Nelson WG, DeWeese TL and DeMarzo AM: The diet, prostate inflammation, and the development of prostate cancer. Cancer Metastasis Rev 21: 3-16, 2002.

47. Bartel DP: MicroRNAs: genomics, biogenesis, mechanism, and function. Cell 116: 281-297, 2004.

48. Lin SL, Chiang A, Chang D and Ying SY: Loss of mir-146a function in hormone-refractory prostate cancer. RNA 14 417-424, 2008.

49. Musiyenko A, Bitko V and Barik S: Ectopic expression of miR-126*, an intronic product of the vascular endothelial EGF-like 7 gene, regulates protein translation and invasiveness of prostate cancer LNCaP cells. J Mol Med 86: 313-322, 2008.

50. Varambally S, Cao Q, Mani RS, et al: Genomic loss of microRNA101 leads to overexpression of histone methyltransferase EZH2 in cancer. Science 322: 1695-1699, 2008.

51. Kumar-Sinha C, Tomlins SA and Chinnaiyan AM: Recurrent gene fusions in prostate cancer. Nature Rev 8: 497-511, 2008

52. He J, Pan Y, Hu J, Albarracin C, Wu Y and Dai JL: Profile of Ets gene expression in human breast carcinoma. Cancer Biol Ther 6: 76-82, 2007.

53. Tomlins SA, Rhodes DR, Perner S, et al: Recurrent fusion of TMPRSS2 and ETS transcription factor genes in prostate cancer. Science 310: 644-648, 2005.

54. Furusato B, Tan SH, Young D, et al: ERG oncoprotein expression in prostate cancer: clonal progression of ERG-positive tumor cells and potential for ERG-based stratification. Prostate Cancer Prostatic Dis 13: 228-237, 2010.

55. Watson D, Turner D, Scheiber M, Findlay V and Watson P: ETS transcription factor expression and conversion during prostate and breast cancer progression. Open Cancer J 3: 24-29, 2010.

56. Okuducu AF, Hahne JC, Von Deimling A and Wernert N Laser-assisted microdissection, techniques and applications in pathology (Review). Int J Mol Med 15: 763-769, 2005.

57. Okuducu AF, Janzen V, Hahne JC, Ko Y and Wernert N: Influence of histochemical stains on quantitative gene expression analysis after laser-assisted microdissection. Int $\mathbf{J}$ Mol Med 11: 449-453, 2003

58. Wellmann A, Wollscheid V, Lu H, et al: Analysis of microdissected prostate tissue with ProteinChip arrays: A way to new insights into carcinogenesis and to diagnostic tools. Int $\mathrm{J}$ Mol Med 9: 341-347, 2002

59. Kohn EC: Invasion and metastasis: biology and clinical potential. Pharmacol Ther 52: 235-244, 1991.

60. Duffy MJ, McGowan PM and Gallagher WM: Cancer invasion and metastasis: changing views. J Pathol 214: 283-293, 2008

61. Duffy MJ: Cancer metastasis: biological and clinical aspects. Ir J Med Sci 167: 4-8, 1998

62. Varga AE, Stourman NV, Zheng Q, et al: Silencing of the Tropomyosin-1 gene by DNA methylation alters tumor suppressor function of TGF-beta. Oncogene 24: 5043-5052, 2005.

63. Bandyopadhyay S, Pai SK, Hirota S, et al: PTEN up-regulates the tumor metastasis suppressor gene Drg-1 in prostate and breast cancer. Cancer Res 64: 7655-7660, 2004.
64. Kamb A, Gruis NA, Weaver-Feldhaus J, et al: A cell cycle regulator potentially involved in genesis of many tumor types. Science 264: 436-440, 1994.

65. Borg A, Sandberg T, Nilsson K, et al: High frequency of multiple melanomas and breast and pancreas carcinomas in CDKN2A mutation-positive melanoma families. J Natl Cancer Inst 92: 1260-1266, 2000.

66. Castellano M, Pollock PM, Walters MK, et al: CDKN2A/p16 is inactivated in most melanoma cell lines. Cancer Res 57: 4868-4875, 1997.

67. Cobbers JM, Wolter M, Reifenberger J, et al: Frequent inactivation of CDKN2A and rare mutation of TP53 in PCNSL. Brain Pathol 8: 263-276, 1998.

68. Perez-Stable CM, Pozas A and Roos BA: A role for GATA transcription factors in the androgen regulation of the prostate-specific antigen gene enhancer. Mol Cell Endocrinol 167: 43-53, 2000.

69. Mehra R, Varambally S, Ding L, et al: Identification of GATA3 as a breast cancer prognostic marker by global gene expression meta-analysis. Cancer Res 65: 11259-11264, 2005.

70. Giles RH, Peters DJ and Breuning MH: Conjunction dysfunction: CBP/p300 in human disease. Trends Genet 14: 178-183, 1998.

71. Zutter MM, Mazoujian G and Santoro SA: Decreased expression of integrin adhesive protein receptors in adenocarcinoma of the breast. Am J Pathol 137: 863-870, 1990

72. Enomoto H, Ozaki T, Takahashi E, et al: Identification of human DAN gene, mapping to the putative neuroblastoma tumor suppressor locus. Oncogene 9: 2785-2791, 1994.

73. Asmann YW, Kosari F, Wang K, Cheville JC and Vasmatzis G: Identification of differentially expressed genes in normal and malignant prostate by electronic profiling of expressed sequence tags. Cancer Res 62: 3308-3314, 2002.

74. Dubbink HJ, Verkaik NS, Faber PW, Trapman J, Schroder FH and Romijn JC: Tissue specific and androgen-regulated expression of human prostate-specific transglutaminase. Biochem J 315: 901-908, 1996.

75. Thielen JL, Volzing KG, Collier LS, Green LE, Largaespada DA and Marker PC: Markers of prostate region-specific epithelial identity define anatomical locations in the mouse prostate that are molecularly similar to human prostate cancers. Differentiation 75: 49-61, 2007.

76. Garraway IP, Seligson D, Said J, Horvath S and Reiter RE: Trefoil factor 3 is overexpressed in human prostate cancer. Prostate 61: 209-214, 2004.

77. Rickman DS, Chen YB, Banerjee S, et al: ERG cooperates with androgen receptor in regulating trefoil factor 3 in prostate cancer disease progression. Neoplasia 12: 1031-1040, 2010.

78. Vaarala MH, Porvari K, Kyllonen A, Lukkarinen O and Vihko P: The TMPRSS2 gene encoding transmembrane serine protease is overexpressed in a majority of prostate cancer patients: detection of mutated TMPRSS2 form in a case of aggressive disease. Int J Cancer 94: 705-710, 2001. 